\title{
Person Identification And Healthy Monitoring Based On Biometric Signal
}

\author{
Nurul Syazana Ismail ${ }^{1}$, Haryati Jaafar ${ }^{2}$,Nur Hidayah Ramli ${ }^{3}$, Aimi Salihah Abdul Nasir ${ }^{4}$ \\ \{haryati@unimap.edu.my ${ }^{1}$, hidayahramli@unimap.edu.my ${ }^{3}$, aimisalihah@unimap.edu.my ${ }^{4}$ \} \\ 1,2,3,4Faculty of Engineering Technology, Universiti Malaysia Perlis, UniCITI Alam Campus, Sg. Chuchuh, \\ 02100 Padang Besar, Perlis, Malaysia
}

\begin{abstract}
In security systems, human identification has an important role. Since, an individual's ECG signals cannot be faked, heartbeat can be used as modality in the biometric system. Aside the security system, another problem that rises is the health of heart condition. The healthy monitoring should be considered as well since the heart attack is the third major of caused dead in world. Thus, this research proposed, person identification and healthy monitoring based on biometric signal which can function as biometrics authentication system. The system was divided into two parts which were hardware implementation and software implementation. In hardware implementation, a heartbeat detection kit and healthy monitoring kit were designed. This kits encompasses of microcontroller and sensors to determine the ECG signal and heart rate pulse based on conditions. The software implementation involves with data collection, feature extraction and classification process. Beside, ECG detection kit was developed for personal system $\log$ in or access control and healthy monitoring kit can monitor health progress of people. The result was achieved with $95.83 \%$ was obtained.
\end{abstract}

Keywords: ECG signal, signal segmentation, feature extraction, classification.

\section{Introduction}

In security systems, human identification has an important role. Biometrics is the science of authenticating and identifying individuals based on people's physical such as palm print, fingerprint, face, iris or using some aspect behavioral characteristics such as voice, signature, gesture and electrocardiographic (ECG) signals (Jain et al., 2004); (Mir et al., 2011). The heart is the organ that in charge of pumping blood all through the body. It is situated amidst the thorax, somewhat counterbalanced to one side and encompassed by the lungs. Because of a person's ECG signals can't be faked, heartbeat can be utilized as modality in the biometric system (Sidek et al., 2014).

Recently, consideration has been attracted the work of another biometric quality, the ECG. ECG signal reflect the heart electrical movement and in this way, have been examined for medical diagnostic purposes completely. Identifying subjects with the ECG is moderately youthful however it grasps impressive points of interest.Basically, health of person can be identified based on the rate of heartbeat. So, healthy monitoring by using heartbeat rate can provide progress of health system of a person. This might help in detecting any disease or health problems based on heartbeat rate.

In recent time, some studies based on ECG signal have been applied. Although such devices are useful, the cost is usually high and beyond the reach of individuals. In addition, the research for ECG based biometric recognition is in its infant phase. These previous studies were implemented to the healthy subjects that considered has a regular heartbeat.However, majority of studies ignored the variability of the ECG during life 
span. In real life, the heartbeat and healthy heart does not have by everyone due to the effect of lifestyles. Even though, on this matter have been initial investigations, but the area is still under research and a lot of justification is needed to prove the robustness of ECG biometric in heartbeat conditions. Moreover, some research concentrate on certain kinds of heart diseases.

Another problem encountered is; nowadays people more materialistic which they run for wealthy without taking care of their health. Therefore, according to World Health Organization (WHO), the third highest cause of dead is cardiovascular disease (CVD). Therefore, it is important to monitor the heart rate frequently such that the problem of CVD can be reduced.

This study investigates the possibility and effectiveness of heartbeat signal for biometric recognition while monitoring the health condition of user. This can save time, money and energy as well, because they can monitor their health progress without go and meet health consultant.

\section{Methodology}

The system is divided into two which are person identification and healthy monitoring. The operation of project consists of two main parts which are hardware implementation and software implementation.

\subsection{Hardware Implementation}

In the project, Arduino Uno R3 is selected as a microcontroller. For person identification, the heartbeat sensor is connected to AD8232 heart monitor as shown in Figure 1. The AD8232 SparkFun Single Lead Heart Rate Monitor is a cost-effective board used to measure the electrical activity of the heart. This electrical activity can be charted as an ECG or Electrocardiogram and output as an analog reading. Since the ECG signal can be extremely noisy, the AD8232 Single Lead Heart Rate Monitor acts as an op amp to help obtain a clear signal from the PR and QT intervals easily.

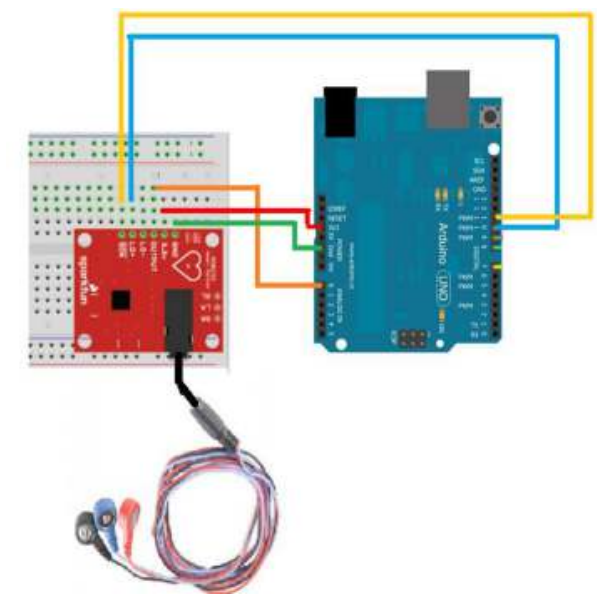

Fig.1. The complete simulation circuit for person identification 
On the others hand, the pulse sensor is applied for healthy monitoring purpose. This sensor amped is a plug and play heart rate sensor for Arduino as shown in Figure 2. It essentially combines a simple optical heart rate sensor with amplification and noise cancellation circuitry making it fast and easy to get reliable pulse readings. Also, it sips power with just $4 \mathrm{~mA}$ current draw at $5 \mathrm{~V}$. The heart rate is read when pulse sensor is placed to fingertip and it is plugged into 5 Volt Arduino. Based on the data from sensor, Arduino calculated the heart rate and displayed the heart rate in beat per minute (bpm).

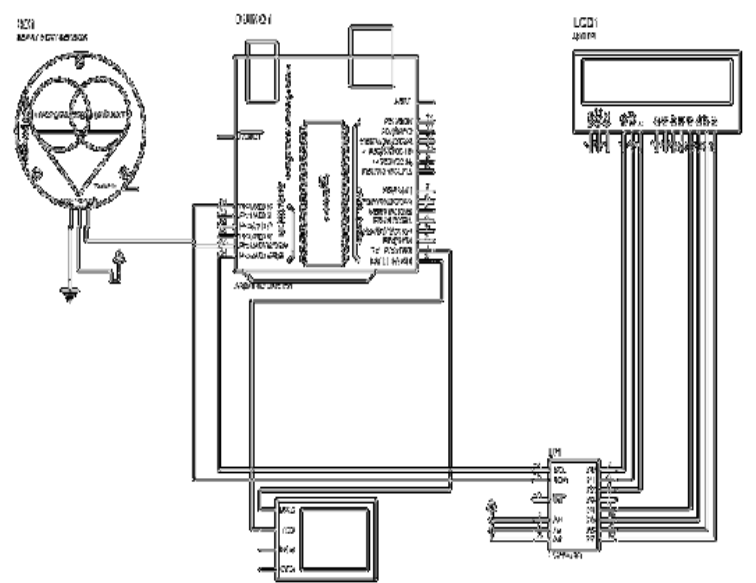

Fig.2. The complete simulation circuit for healthy monitoring

\subsection{Software Implementation}

\subsubsection{Data acquisition}

In order to determine the person identification based on ECG signal, the signal is determined when the user was in normal conditions. Users were required to remain calm and relaxed throughout the recording session. A total of 2 healthy users with different age were participated in this systems which 22 and 45 years old. This is because different age people have different health condition. So that, the system was tested on this two people to prove that this system able to detect health condition no matter old or young of the person. The cable of heartbeat sensor is labelled to help identify proper placement. Figure 3 shows the typical sensor placement. The data have been recorded five times for each of the users to get the accurate data. The signal length for each ECG signal is approximated $0.5 \mathrm{~ms}$. Therefore, the frequency sampling (fs) of $16 \mathrm{kHz}$ was applied in this process.

For healthy monitoring system, in order to determine the suitable frequency for different types of heartbeat condition, a study based on bpm has been employed. The relationship of heartbeat and frequency is depicted in Equation 1 and the suitable frequency for different condition is presented in Table 1. 


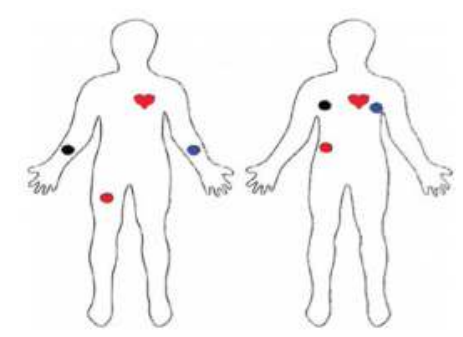

Fig. 3. Typical sensor placement

$f=\frac{1}{T}=\frac{b p m}{60}$

where $f$ is the suitable frequency and $T$ represents one period of cycle.

Table 1. The suitable frequency for different condition

\begin{tabular}{ll}
\hline Condition & Frequency $(\mathrm{Hz})$ \\
\hline $\begin{array}{l}\text { Rest } \\
(40-59 \text { bpm })\end{array}$ & 1.17 \\
\hline $\begin{array}{l}\text { Before physical activities } \\
(60-99 \text { bpm })\end{array}$ & 2.17 \\
\hline $\begin{array}{l}\text { After physical activities } \\
(100-170 \text { bpm })\end{array}$ & 0.75 \\
\hline
\end{tabular}

\subsubsection{Pre-processing}

Each of the data undergoes a series of speech processing step that is preemphasis, framing and windowingThe pre-emphasis process is the process to compress the signal dynamic range by passing it through a filter to emphasize the signal to higher frequencies in order to raise Signal to Noise Ratio (SNR) (Ramli et al., 2016).In this process, the speech signal was filtered with the first order FIR filter whose transfer functions in the z-domain as given as;

$H(z)=1-\alpha z^{-1} \quad 0 \leq \alpha \leq 1$

In essence, a pre-emphasis filter in time domain is a first order high pass-filter;

$X^{\prime}(n)=x(n)-\alpha x(n-1)$

where $\mathrm{a}$ is the pre-emphasis parameter. A typical value for $\mathrm{a}$ is specifying as 0.95

In this study, Hamming window most frequently applied since high resolution is not required, whose impulse response is a raised cosine impulse and defined as;

$W(n)=0.54-0.46 \cos (2 \pi n / N-1), n=0, \ldots, N-1$ 


\subsubsection{Signal Segmentation}

The raw ECGs signal is needed to go through the de noised process before the segmentation takes places because the raw signals are often contaminated with noises. After filtering, each individual heartbeat waveform will be segmented from the full recording. Then, two techniques was applied based on time-domain which are the Short Time Energy (STE) and Short Time Average Zero Crossing Rate (STAZCR) (Jaafar et al., 2013).

In the STE technique, energy of a call is another parameter for classifying sound/unsound parts. The voiced part has high energy than unvoiced part due to the periodicity. The STE function applied for the recorded signal is defined by the following expression;

$E_{n}=\frac{1}{N} \sum_{m=1}^{N}[x(m) w(n-m)]^{2}$

where $E_{n}$ is the energy of the sample n of the signal, $x(m)$ is the discrete-time signal and $w[m]$ is a hamming window of size $N$.

On the other hands, STAZCR is often used as a part of the front-end processing in automatic speech recognition system. During the frog signal processing, the amplitude of the unvoiced part normally have much higher values and vice- versa. The ZCR is the rate at which signal changes from positive to negative and back and defined as;

$Z_{n}=\frac{1}{2 N} \sum_{m=1}^{N}|\operatorname{sgn} x(m)-\operatorname{sgn}[x(m-1)]| w(n-m)(6)$

where

$\operatorname{sgn}[x(m)]=\left\{\begin{array}{c}1, x(m) \geq 0 \\ -1, x(m)<0\end{array}\right.$

Figure 4 illustrates the outputs of the segmentation by using the STE and STAZCR techniques.

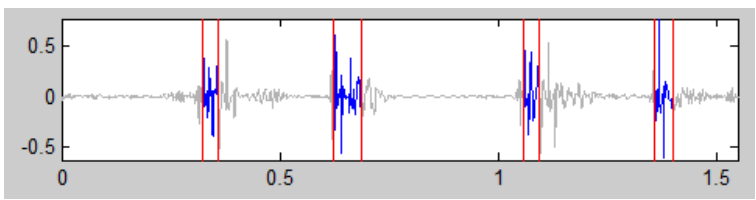

Fig. 4. The outputs of the segmentation

\subsubsection{Feature Extraction}

MFCC is selected due to the features are robust to noise. The operation of this system is based on two types of filter which are linearly and logarithmically spaced and processes on the Fourier transform of $x_{t}(n)$ : $X_{t}\left(e^{j \omega}\right)$. The $X_{t}\left(e^{j \omega}\right)$ is evaluated only for discrete number of $\omega$ values (Hassan et al., 2012). 
There have several steps in MFCC processing. The first step is computation of the Discrete Fourier Transform (DFT) of all frames of the signal. By considering $\omega=2 \pi \mathrm{k} / \mathrm{N}$, the DFT of all frames of the signal, $\mathrm{x}_{\mathrm{t}}(\mathrm{k})$ is obtained as:

$x_{t}(k): X_{t}\left(e^{j 2 \pi k} / N\right), k=0, \ldots, N-1$

The computational complexity can also be reduced if the number of samples $\mathrm{N}$ is a power of 2. The result obtained after this step is called as signal's spectrum.

A filter bank processing is the second step in MFCC processing. Filter banks properly integrate a spectrum at defined frequency and spectral features are obtained after this process. The outputs of the filter bank are denoted as $Y_{t}(m), 1 \leq m \leq M$ where $\mathrm{M}$ is number of band-pass filters. In general, a set of 24 band-pass filter is used. Subsequently, computation of the log energy is the third step which computes the logarithm of the square magnitude of the filter banks outputs, $y_{t}(m)$ (Chen et al., 2009; Somervuo et al., 2006). The final step for MFCC processing is mel frequency cepstrum computation that performs the inverse DFT on the logarithm of the magnitude of the filter bank output:

$y_{t}^{m}(k)=\sum_{m=1}^{M} \log \left\{Y_{t}(m)\right\} \cdot \cos (k(m-0.5)(\pi / M))(9)$

In this study, the database of MFCC features consists of 24 set of MFCC features from 2 persons with 12 signals data per person. There are 12 mel cepstrum coefficients, one log energy coefficients per frame. The signal is divided into overlapping frames of 256 samples with $50 \%$ overlap.

\subsubsection{Classification}

Nearest neighbour technique is a nonparametric classifier that employs lazy learning and has been practiced in various sound analysis (Jaafar et al., 2016). Given a set of parameters, $\mathrm{kNN}$ classifier will find the nearest neighbour among training data by determining the minimum distance between query instance (testing set) and each training set. In the absence of prior knowledge, most kNN classifier use Euclidean distance with $d_{E}(x, y)$ to measure the distance or similarity between query instance and training set.

$$
d_{E}=\left(x_{i}^{2}-y_{i}^{2}\right)^{0.5}
$$

where $x, y$ are training and testing samples composed of feature $N$, respectively.

Each query instance will be compared with training set. A classification combination method that combines the selected training set and the query instance is then applied. The simplest classification method is the voting method where the class label of the query instance is determined based on the majority voting among the $\mathrm{k}$ nearest training samples category.

The $\mathrm{k}$ values represent an important role in kNN classification. Generally, kvalues of $\mathrm{kNN}$ should be determined in advance and the best choice of $\mathrm{k}$-values 
depends on the data. Normally, larger values of $\mathrm{k}$ will reduce the effect of noise on the classification but cause boundaries between classes less distinct.

\subsection{Graphic User Interface (GUI) Implementation}

In this study, Matlab programming is used to develop the system for healthy monitoring for person identification. To run the GUI system simulation, the Arduino must be connected with Matlab software for the heartbeat monitoring systems. Figure 5 shows overall systems displayed on GUI. For healthy monitoring, when the heartbeat push button is pressed, the heart rate pulse sensor start to count the pulse and the condition of the user will be displayed. Then, for person identification, when the load push button is pressed the ECGs signals of the user displayed on GUI systems. When the recognition push button is pressed, the systems will detect the owner of the users.

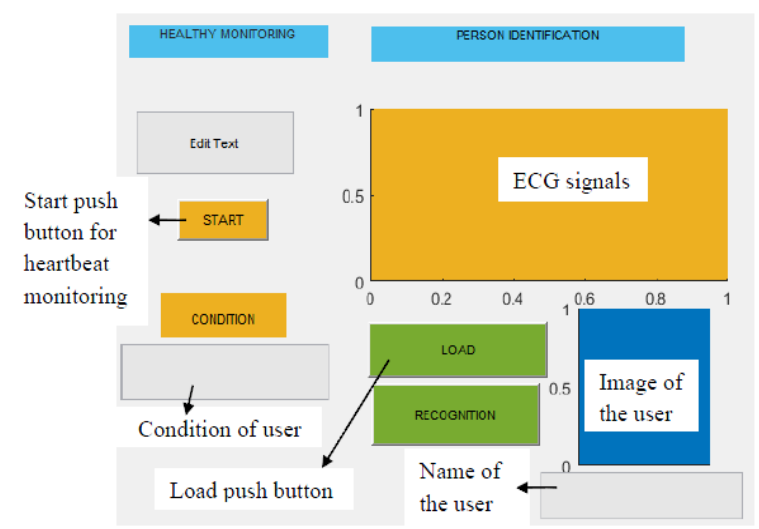

Fig. 5 Overall system displayed on GUI

An error could occur if incorrect data is inserted in the system. This will prompt an error window appears as shown in Figure 6. The warning window will prompt to alert the user to input the data correctly in the system.

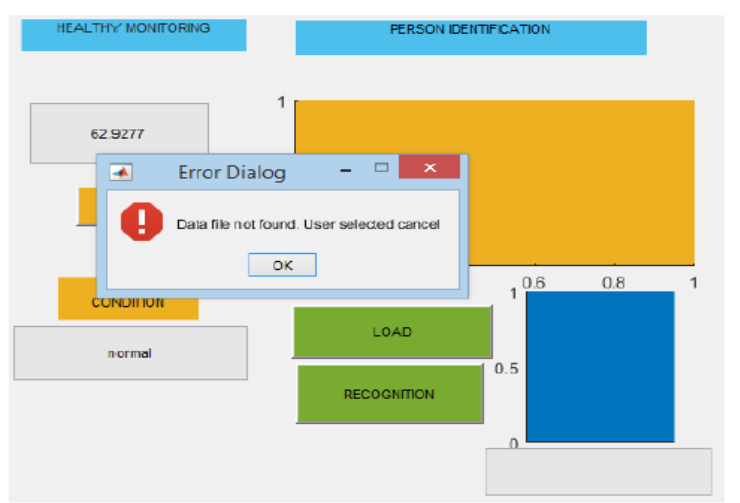

Fig. 6. The error detection to make necessary adjustments 


\section{Experimental Results}

The experiments are implemented using Matlab R2017(b) and have been test in Intel Core i5, 2.1GHz CPU, 2G RAM and Window 7 operating system.

\subsection{Signal segmentation for GUI}

Figure 7(a) shows the signal before segmentation process for GUI. The result of segmentation can be seen as shown in Figure 7(b).

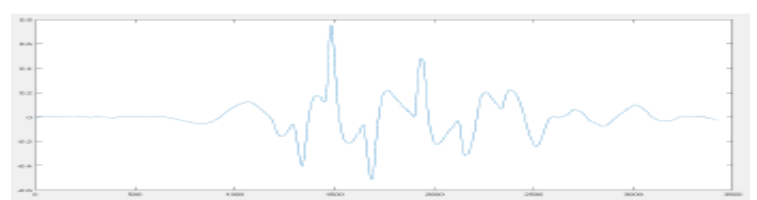

(a) Signal before segmentation process

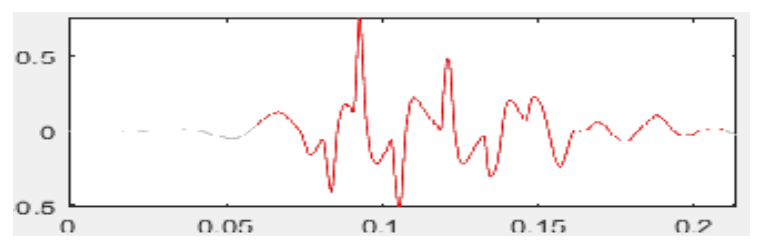

(b) Signal after segmentation process

(c)

Fig. 7. Signal segmentation process

From the Figure 7, it shows that the STE and STAZCR able to segment the signals in different condition. The STE and ZCR was able to provide good segmentation results.

\subsection{Performance of Classification}

\subsubsection{Performance of Classification Based On Different Value of $\boldsymbol{k}$}

In order to identify the person based on ECG signal, a template matching is compared by two data which are called the enrolment or training and recognition or testing data. Table 4.1 indicates the accuracy for matching process. For studying the computational efficiency, the data were extracted by using MFCC and the dimension was fixed at 4096. The value of $\mathrm{k}$ were set in the odd numbers ranging from 1 to 15 for the kNN classifier. The performance is evaluated based on classification accuracy (CA).

Table 2 shows that the value $k=1$ was performed since the accuracy is $100 \%$. However, value $k=3$ was used in this project to avoid to gain an unbiased estimation of an accuracy generalization. The results was performed by employing the CA rate and processing time.

Table 2: The accuracy for matching process

\begin{tabular}{ccc}
\hline $\mathbf{k}$ & Accuracy (\%) & Time Processing (s) \\
\hline 1 & 100.0000 & 0.1975 \\
\hline 3 & 95.8333 & 0.1419 \\
\hline 5 & 91.6667 & 0.0911 \\
\hline 7 & 83.3333 & 0.0977 \\
\hline 9 & 83.3333 & 0.1013 \\
\hline
\end{tabular}




\begin{tabular}{lll}
\hline 11 & 83.3333 & 0.1061 \\
\hline 13 & 75.0000 & 0.1043 \\
\hline 15 & 75.0000 & 0.0943 \\
\hline
\end{tabular}

\subsubsection{Performance of Classification Based on GUI}

For person identification, the offline system was developed due to the electrode of ECG sensor has to be placed properly to the part of body. Here, two users involved in this experiment. Figure 8(a) shows the input before the recognition process and Figure 8(b) presents the snapshot's result of the GUI for person identification.

For healthy monitoring, the online system was developed. Three condition was set to the system (rest, before physical activities (normal) and after physical activities). The user is asked to place their fingertip to the sensor in different condition which are rest, before physical activities (normal) and after physical activities. The result is determined based on beat per minute (bpm) that displayed in GUI. For example when the bpm of user is around 40-59 bpm the system will be declared as rest condition (Figure 9).

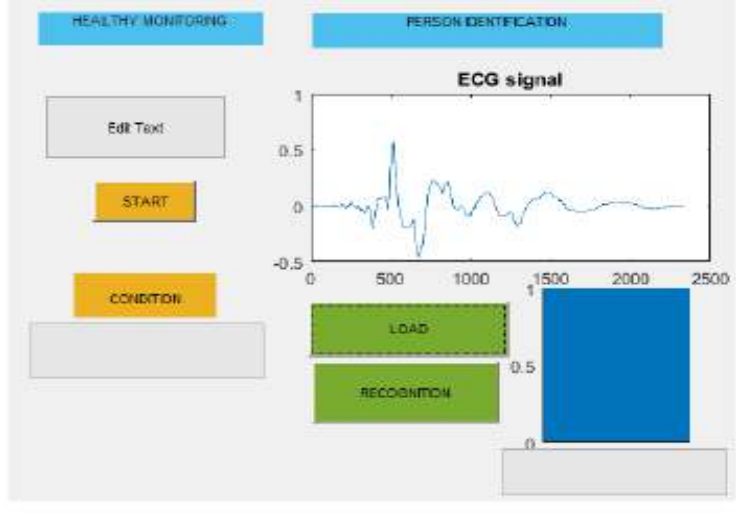

(a) Before recognition

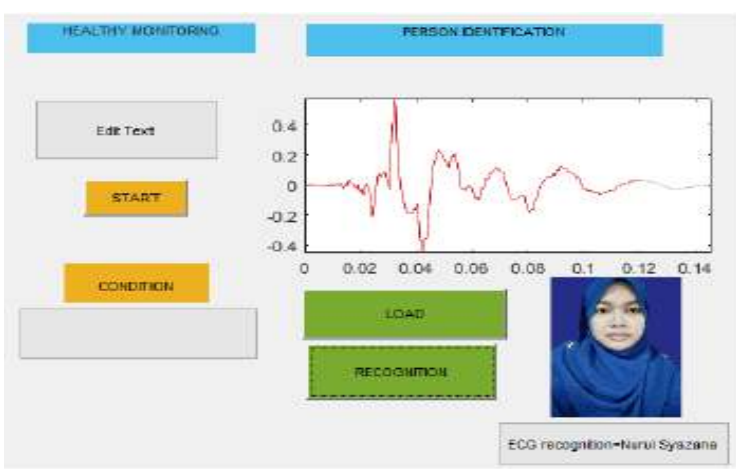

(b) After recognition

Fig. 8. Person identification system 


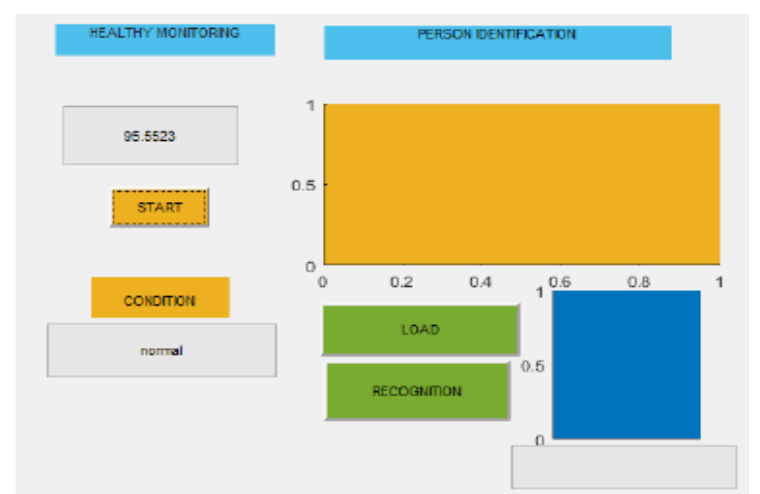

Fig. 9. Health monitoring system

\section{Conclusions}

In this study, the system for person identification and healthy monitoring based on biometric signalhas been proposed. This system involves with the hardware implementation, data acquisition and software implementation. For person identification, the heartbeat conditions are based on regular (rest, normal) and irregular (physical activities). It can be concluded that the person identification is successfully identify the user in with more than $95 \%$. Meanwhile, the healthy monitoring is successfully implemented in GUI Matlab programming where three conditions; rest, before physical activities (normal) and after physical activities is detected.

\section{Acknowledgements}

The authors would like to express their gratitude for the financial support provided by Universiti Malaysia Perlis Short Term Grant (9001-00526).

\section{References}

[1] Chen, S. H., Luo, Y. R., 2009. Speaker verification using MFCC and support vector machine,Proceedings of the International MultiConference of Engineers and Computer Scientists (IMECS 2009), vol. 1, pp. 532-535.

[2] Hasan, M.H., Jaafar, H., Ramli. D.A. 2012. Evaluation on score reliability for biometric speaker authentication system, Journal of Computer Sciences, vol. 8, no.9, pp. 1554-1563.

[3] Jaafar, H., Mukahar, N., Ramli, D.A., 2016. A methodology of nearest neighbor: Design and comparison of biometric image database,IEEE Student Conference on Research and Development (SCOReD), pp 1-6.

[4] Jaafar, H., Ramli, D.A., 2013. Automatic syllables segmentation for frog identification system, in 2013 IEEE Int. Colloquium on Signal Processing and its App, vol. 9.

[5] Jain, A. R., Prabhakar, S. 2004. An Introduction to Biometric Recognition. Transactions on Circuits and Systems for Video Technology, 4-20.

[6] Mir, A. R., Jhat, Z. 2011. Biometric Verification: A Literature Survey. Journal of Computing \& ICT Research, 67-80. 
[7] Ramli, D.A., Jaafar, H. 2016. Peak Finding Algorithm to Improve Syllable Segmentation for Noisy Bioacoustic Sound Signal, Procedia Computer Science, vol. 96, pp.100-109.

[8] Sidek K. A., Mai V., Khalil, I. 2014. Data mining in mobile ECG based biometric identification, Journal of Network and Computer Applications, vol. 44, pp. 83-91.

[9] Somervuo, P., Harma, A., Fagerlund, S., 2006. Parametric representations of bird sounds for automatic species recognition,IEEE Transactions Audio, Speech, and Language Processing, vol. 14, pp. 2252-2263. 